\title{
Interrelación Entre Ortodoncia y Periodoncia en Periodonto Reducido. Estabilidad Periodontal Tras 6 Años de Seguimiento.
}

\section{Interrelation Between Orthodontics and Periodontics in Reduced Periodontium. Periodontal Stability After 6 Years of Follow-up.}

\author{
Cristián Navarrete ${ }^{1,2^{\star}}$, Ma. Angélica Michea ${ }^{2,3}$, Hans von Müllenbrock ${ }^{1,2}$, Claudia González ${ }^{1,2}$, \\ Ru-Harn Chang ${ }^{4}$, Ada Pango ${ }^{5}$, René Lobo ${ }^{6}$, Alejandro Riquelme ${ }^{2,3}$
}

\author{
1. Departamento del Niño y Ortopedia \\ Dentomaxilar, Facultad de Odontología, \\ Universidad de Chile, Santiago, Chile. \\ 2. Unidad Ortodoncia - Periodoncia, Facultad de \\ Odontología Universidad de Chile, Santiago, Chile. \\ 3. Departamento de Odontología Conservadora, \\ Facultad de Odontología, Universidad de Chile, \\ Santiago, Chile. \\ 4. Servicio de Salud de Aysén, Chile \\ 5. Universidad de Nápoles Federico II, Nápoles, \\ Italia \\ 6. Práctica Privada \\ * Correspondencia Autor: Cristián Navarrete \\ Contreras. | Dirección: Sergio Livingstone P 943 \\ Independencia (8380492). Santiago de Chile | \\ Teléfono +56229781839 | E-mail: cnavarrete@ \\ odontologia.uchile.cl \\ Trabajo recibido el 29/04/2020. \\ Trabajo revisado 09/08/2020 \\ Aprobado para su publicación el 21/08/2020
}

\begin{abstract}
RESUMEN
En pacientes con periodontitis, la migración dentaria patológica es una consecuencia de la pérdida de soporte periodontal. El siguiente reporte de caso aborda el tratamiento periodontal y ortodóncico de una paciente con periodontitis y migración dentaria patológica. El protocolo utilizado fue el protocolo de la Unidad de Ortodoncia y Periodoncia de la Facultad de Odontología de la Universidad de Chile. La estabilidad periodontal se ha mantenido por al menos 6 años luego de finalizado el tratamiento de ortodoncia logran-do funcionalidad y estética de la dentición natural.
\end{abstract}

PALABRAS CLAVE:

Migración dental; Periodontitis; Técnicas de movimiento ortodóncico.

Int. J. Inter. Dent Vol. 14(3); 278-281, 2021.

\section{ABSTRACT}

In patients with periodontitis, pathologic tooth migration is a consequence of loss of periodontal support. The following case report addresses the periodontal and orthodontic treatment of a patient with periodontitis and pathologic tooth migration. The protocol used was the protocol of the Orthodontics and Periodontics Unit of the Faculty of Dentistry of the University of Chile. The periodontal stability has been maintained for at least 6 years after the end of orthodontic treatment, achieving functionality and aesthetics of the natural dentition.

KEY WORDS:

Tooth migration; Periodontitis; Tooth movement techniques.

Int. J. Inter. Dent Vol. 14(3); 278-281, 2021.

\section{INTRODUCCIÓN}

Dentro de las secuelas de la periodontitis se puede encontrar la migración dentaria patológica (MDP). Ésta se define como el cambio de la posición inicial de un diente producto de la disrupción de las fuerzas que lo mantienen en posición ${ }^{(1)}$. Dentro de los diferentes movimientos ortodóncicos realizados en los pacientes con periodonto reducido producto de periodontitis, la intrusión ortodóncica es un movimiento ejecutado frecuentemente para corregir la extrusión dentaria que presentan los pacientes como consecuencia de la pérdida de soporte periodontal ${ }^{(2)}$. La intrusión ortodóncica corresponde al desplazamiento del diente en dirección apical y es una técnica de movimiento ortodóncico controversial ya que puede presentar como efecto no deseado la reabsorción radicular apical externa ${ }^{(3)}$. Se considera aceptable de 1 a $3 \mathrm{~mm}$. de reabsorción radicular apical externa cuando ésta no ha comprometido la vitalidad pulpar ${ }^{(4)}$. En ausencia de un adecuado control del biofilm dental se ha demostrado la formación de sacos periodontales infraóseos durante la intrusión dental ${ }^{(5)}$, sin embargo, en presencia de buen control de biofilm dental durante todo el tratamiento de ortodoncia (TO) existe menor inflamación gingival, ausencia de pérdida de inserción clínica e inclusive se ha descrito signos de aposición ósea en el hueso marginal( ${ }^{(6)}$. Así, la mantención de una buena higiene oral durante todo el tratamiento periodontal (TP) y TO es un punto clave para evitar la reabsorción ósea adicional y la formación de sacos periodontales. Así mismo, se ha observado que la intrusión de los dientes no resulta en una disminución del nivel de hueso marginal cuando la inflamación gingival está controlada ${ }^{(7)}$. Se debe hacer hincapié durante todo el tratamiento que un adecuado control de biofilm dental es necesario para lograr la estabilidad periodontal y el paciente debe comprender los objetivos del tratamiento para poder abordar su condición desde un punto de vista interdisciplinario, apoyándose de la periodoncia, ortodoncia y de otras especialidades. El presente caso clínico tiene como objetivo mostrar el TP, TO y tratamiento rehabilitador mediante un implante dental de una paciente con periodontitis y MDP, donde la modalidad de tratamiento con el protocolo de la Unidad de Ortodoncia y Periodoncia de la Facultad de Odontología de la Universidad de Chile (FOUCH), ha permitido corregir la malposición dental y mantener la dentición natural por al menos 6 años luego de finalizado el TO.

\section{INFORME DEL CASO}

Paciente género femenino, 64 años, sin antecedentes médicos. Tratada entre los años 2010 y 2020. Su motivo de consulta en FOUCH es que sus dientes anterosuperiores se han ido separando y uno de ellos lo ve más "largo y suelto". Relata que había recibido TP recientemente, donde se le había sugerido realizar la exodoncia de diente 1.1 y su reemplazo por un implante dental. La paciente deseaba un tratamiento que considerara corregir la malposición dental y la mantención de 
su dentición. Al examen periodontal se observa pérdida de inserción periodontal generalizada y MDP en sextante 2 y 5 (Figura 1). Se realiza periodontograma inicial (Figura 2a), en el cual se pesquisa un $66 \%$ de sitios con biofilm dental, sangrado al sondaje de $43 \%$, múltiples recesiones gingivales y una pérdida del nivel de inserción clínica (NIC) en promedio de $3,7 \mathrm{~mm}$. maxilar y mandibular. Los dientes más comprometidos presentaban un NIC de $9 \mathrm{~mm}$. en incisivos. Se observó lesión de furca clase I en molares, supuración en los dientes 1.1 y 2.1 y movilidad grado 1, 2 ó 3 en todos los dientes presentes.

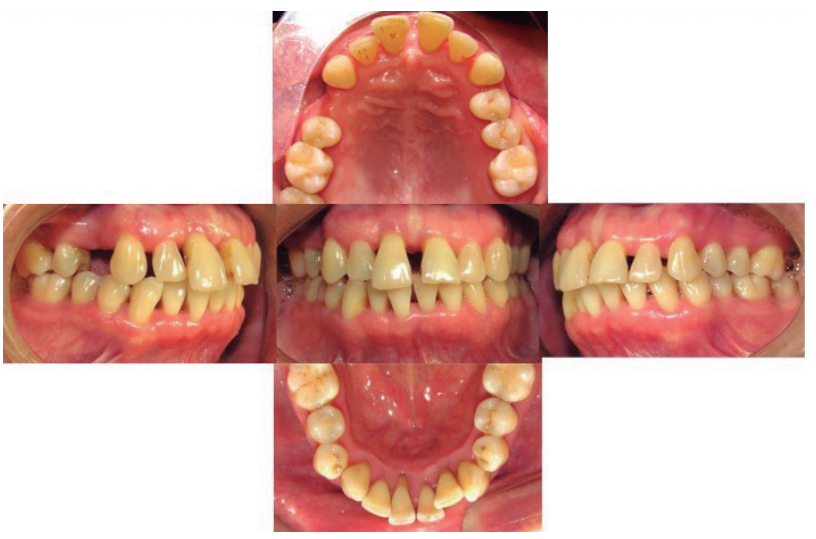

Figura 1. Fotografías intraorales iniciales. Se observa migración dentaria patológica en sextante 2 y 5 con formación de diastemas. Extrusión de diente 1.1

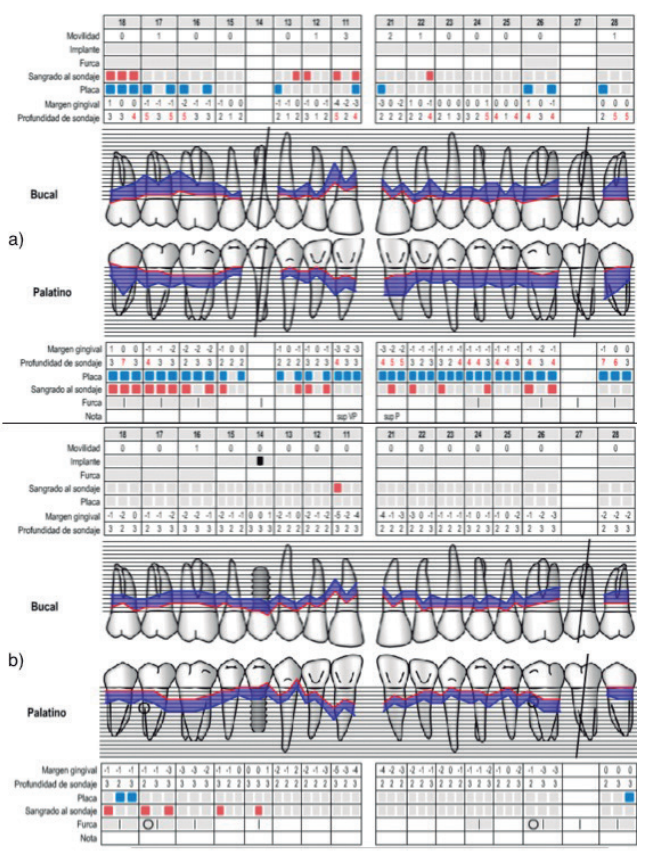

Figura 2. a) Periodontograma maxilar inicial año 2010. b) Periodontograma maxilar de TSP año 2019. Implante dental diente 1.4

Análisis radiográfico: En las radiografías panorámica y periapical de sextante 2 y 5 se observa en el maxilar reabsorción ósea marginal horizontal discreta a marcada generalizada, avanzada en dientes $1.1 \mathrm{y}$ 2.1, en mandíbula se observa reabsorción marginal horizontal discreta generalizada, marcada en incisivos (Figura 3a y $3 b$ ).

Diagnóstico: Paciente con periodontitis etapa III generalizada grado $\mathrm{B}$. Tiene MDP en sextante 2 y 5 , proinclinación de dientes 1.1 y 2.1 , con presencia de diastemas y extrusión de diente 1.1. Clase II esqueletal, braquifacial, desdentada parcial, ausencia de dientes 1.4 y 2.7, protrusión y proinclinación incisiva (Figura 3c). Neutroclusión canina derecha, mesioclusión canina izquierda, mesioclusión molar bilateral, overjet $3 \mathrm{~mm}$. y overbite $5 \mathrm{~mm}$.

Tratamiento periodontal: La modalidad de tratamiento se presenta en la Tabla 1. El protocolo de tratamiento fue aprobado por el comité

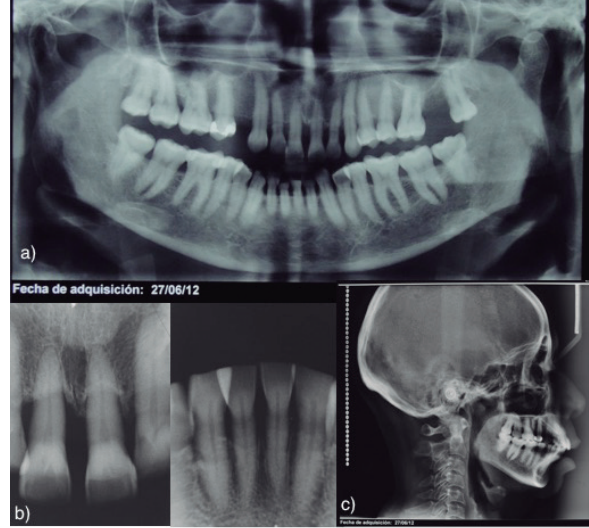

Figura 3. a) Radiografía panorámica al iniciar tratamiento ortodóncico. b) Radiografía retroalveolar dientes 1.1, 2.1 y sextante 5 . c) Teleradiografía de perfil inicial

Tabla 1. Modalidad de tratamiento periodontal FOUCH

\begin{tabular}{|c|c|c|c|}
\hline \multirow{2}{*}{$\begin{array}{r}\text { Fase } \\
\text { Fase Sistémica }\end{array}$} & \multirow{2}{*}{$\begin{array}{l}\text { Descripción } \\
\text { Evaluación de antecedentes médicos. }\end{array}$} & \multicolumn{2}{|c|}{ Duración (sesiones) } \\
\hline & & 1 & 9 meses \\
\hline \multirow[t]{7}{*}{ Fase Etiológica } & $\begin{array}{l}\text { Tratamiento periodontal no quirúrgico: } \\
\text { - Periodontograma inicial } \\
\text { - Enseñanza de etiologia de enfermedad periodontal } \\
\text { - Instrucción de higiene oral } \\
\text { - Destartraje supragingival y subgingival } \\
\text { - Pulido y alisado radicular (por cuadrantes) }\end{array}$ & 6 & \\
\hline & Reevaluación a las 4 semanas, luego del último pulido radicular & 1 & \\
\hline & Retratamiento de sitios inflamados y con cálculos residuales & 1 ó 2 & \\
\hline & $\begin{array}{l}\text { Periodontograma de salida (a las } 12 \text { semanas desde la } \\
\text { reevaluación) }\end{array}$ & 1 & \\
\hline & Retratamiento de sitios inflamados y con cálculos residuales & 1 ó 2 & \\
\hline & Control 1: evaluación del estado periodontal final & 1 & \\
\hline & Control 2: evaluación del estado periodontal final. Alta periodontal & 1 & \\
\hline \multirow[t]{2}{*}{$\begin{array}{l}\text { Terapia de Soporte } \\
\text { Periodontal }\end{array}$} & $\begin{array}{l}\text { - Actualización antecedentes médicos } \\
\text { - Control de Biofilm dental } \\
\text { - Instrucción de higiene oral } \\
\text { - Destartraje supray y subgingival } \\
\text { - Monitorización de surcos profundos ( } \geq 4 \mathrm{~mm}, \text { sin sangrado) }\end{array}$ & \multirow[t]{2}{*}{$\begin{array}{r}\text { Cada } 3 \\
\text { meses } \\
\text { durante } \\
\text { TO }\end{array}$} & \\
\hline & Derivación a Tratamiento de Ortodoncia (TO) & & \\
\hline
\end{tabular}

de ética de $\mathrm{FOUCH}$ y fue realizado bajo consentimiento informado. Se realizó instrucción de higiene oral con técnica de cepillado de barrido con cepillo de filamentos suaves, hilo dental y cepillo interproximal. EI TP fue realizado por especialistas en periodoncia, con curetas periodontales Gracey y Mini-Five, ultrasonido, complementado con terapia antibiótica de amoxicilina $500 \mathrm{mg}$. + metronidazol $250 \mathrm{mg}$. por 7 días al final de los pulidos radiculares. La reevaluación se realizó a las 4 semanas desde el último pulido radicular. Se evaluó inflamación gingival y control de biofilm dental. Luego se citó en 3 meses para realización de periodontograma de salida. Se realizó retratamiento de sitios periodontales que no cumplían los requisitos de salud periodontal en periodonto reducido (sin o mínimo sangrado al sondaje, sin cálculo supra o subgingival, sin sacos periodontales $>5 \mathrm{~mm}$ ). Luego de estar en condición de salud periodontal clínica, se dio el alta periodontal, para posteriormente ser citada a la Terapia de Soporte Periodontal (TSP) cada 3 meses. Luego de dos intervalos de TSP, en donde la paciente mantuvo la estabilidad periodontal obtenida en la fase etiológica, se deriva a TO (Figura 4).

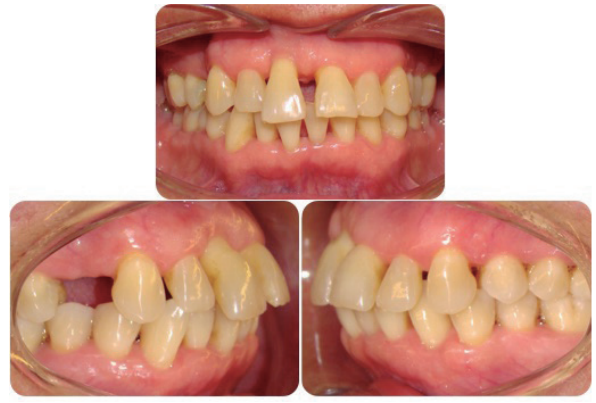

Figura 4. Tejidos periodontales durante TSP 
Tratamiento de ortodoncia: Al finalizar la segunda TSP se inició el TO por especialistas. Se planificó un tratamiento ortodóncico no conservador, con exodoncia del diente 3.1 para solucionar el apiñamiento y proinclinación incisiva inferior, permitiendo corregir el overjet y overbite y adicionalmente, mantener las relaciones oclusales en los sectores laterales. Para ello se utilizaron aparatos fijos prescripción Roth 0,022" (brackets Balance, GAC ${ }^{\circledR}$, EE. UU.) en ambas arcadas, con tubos de cementado directo en molares (Ovation, GAC ${ }^{\circledR}$, EE. UU.). En los incisivos superiores se realizó una cementación estratégica de brackets, posicionándolos más hacia cervical, siguiendo el nivel óseo remanente, observado en la radiografía panorámica. En dientes con pérdida ósea, el centro de resistencia se desplaza apicalmente, lo que determina que al aplicar una fuerza el momento generado sea mayor. La cementación estratégica permite acercar los brackets al centro de resistencia de cada diente y con esto, disminuir el momento que se genera al aplicar una fuerza ortodóncica. En el resto de los dientes, los brackets se cementaron en forma convencional, en el centro de la corona clínica. La paciente fue citada a control cada 4 semanas. En la arcada superior, el primer arco utilizado fue un alambre twist flex 0,0175" con dobleces compensatorios en los incisivos según la posición de los brackets. Una vez corregidas las rotaciones, a los 3 meses de TO, se inicia la intrusión con arco de acero 0,014" llegando como máximo a un acero 0,016 ". En cada control se modificaron gradualmente los dobleces compensatorios (menos de $1 \mathrm{~mm}$.), para lograr la intrusión de las piezas extruídas (Figura 5). El uso de arcos de acero permite entregar fuerzas interrumpidas, que son aquellas que disminuyen a cero entre activaciones, lo que posibilita un período para la reorganización de los tejidos antes de volver a aplicar la fuerza. La alineación y nivelación inferior se obtuvo utilizando arcos de NiTi 0,012" y luego a los tres meses se pasó a un acero 0,014 " por dos meses más. Para la retrusión y cierre de espacio de la exodoncia del incisivo, se usó un arco de acero 0,016" con ansas cerradas distales a 3.2 y 4.2 (Figura 6a), reactivadas control por medio. La fase de finalización, en ambas arcadas, fue obtenida con arcos de acero 0,016" compensados y cinchados. Cabe recalcar, desde un punto de vista ortodóncico, que el objetivo principal en este tipo de pacientes es corregir la migración dentaria patológica, para lo cual se debe mantener un muy buen control de biofilm dental, eliminar las fuerzas oclusales traumáticas, con niveles de fuerza muy bajos y autolimitados, sobretodo en la etapa de intrusión. Luego de 26 meses de tratamiento, se lograron los objetivos del TO y se realizó retiro de aparatos. Se dejó con contención fija en ambas arcadas (alambre twist flex 0,175 "), en incisivos superiores y de canino a canino inferior (Figura 6b), y además con una placa Hawley de asa larga, como contención removible en la arcada superior, de uso día y noche por los primeros seis meses y luego sólo noche hasta completar los dos años de uso. Luego de ello, se fue disminuyendo progresivamente hasta que en el lapso de los dos años subsiguientes se eliminó la contención removible, manteniéndose las contenciones fijas en forma permanente.

Durante todo el TO se mantuvo la TSP en intervalos de 3 meses, controlando cuidadosamente el biofilm dental. Posteriormente, la paciente fue sometida a una cirugía de implante dental para reemplazar el diente 1.4 ausente. Se realizó la evaluación clínica y radiográfica con Cone Beam del sextante 1 con el cual se planificó la posición, inclinación y distancias necesarias con los dientes adyacentes. El implante fue de 4,2 mm. øx $13 \mathrm{~mm}$. sin necesidad de injerto óseo, no se observaron dehiscencias ni fenestraciones del hueso alveolar en el momento de la cirugía, con el cual se obtuvo la estabilidad primaria adecuada con torque de $40 \mathrm{Ncm} 3$. Se esperó un tiempo de oseointegración de 12 semanas y se procedió a la temporización y posterior rehabilitación (Figura 6c). La paciente continuó en TSP por al menos 6 años luego de finalizado el TO, sin historia de infección periodontal o pérdida dentaria por periodontitis.

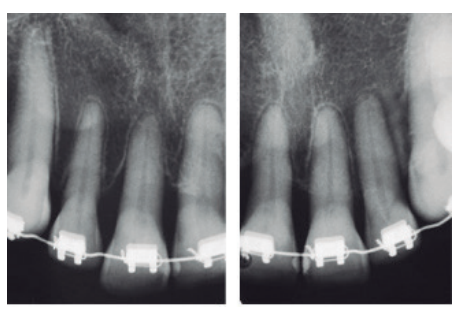

Figura 5. Radiografía retroalveolar sextante 2 a los 13 meses de tratamiento

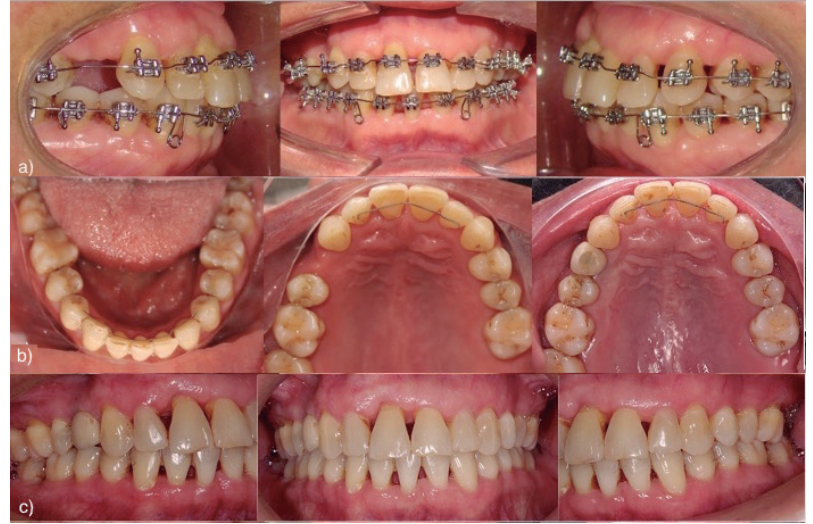

Figura 6. a) 16 meses de tratamiento. b) Contención lingual y palatina c) Tratamiento ortodóncico finalizado. Implante dental diente 1.4

\section{DISCUSIÓN Y CONCLUSIÓN}

Los resultados obtenidos de este caso clínico muestran que la combinación del TP y TO resuelve de modo conservador la MDP, mantiene la salud periodontal lograda al momento del alta periodontal y ésta se mantiene estable durante 6 años posteriores al TO (Figura 2b). EI TP adecuado elimina el componente inflamatorio infeccioso de los tejidos periodontales, permitiendo que el mecanismo inflamatorio aséptico producido por el TO no genere secuelas en los tejidos periodontales ${ }^{(8)}$ teniendo como requisito para que se produzca este mecanismo el óptimo control del biofilm dental durante todo el tratamiento, pese a que los aparatos ortodóncicos acumulan más biofilm en las superficies dentales ${ }^{(9)}$. Lo descrito anteriormente se explicó a la paciente, se le entrenó en una técnica de cepillado eficaz y se le realizó el seguimiento durante las TSP con los aparatos ortodóncicos y luego de haberlos retirado, lo que contribuyó a mantener la salud periodontal. Resultados similares han sido descritos por otros autores ${ }^{(7,10,11,12)}$. En este caso, la medición y el seguimiento de los parámetros periodontales evidencia que la paciente no presenta inflamación periodontal previo, durante y posterior al TO ya que el control de biofilm dental fue reforzado en cada TSP. La mecánica ortodóncica utilizada bajo este protocolo $\mathrm{FOUCH}$ es individualizada ya que la cementación de los brackets es realizada en función del remanente óseo, siendo más apical dentro de la corona clínica en los dientes con periodonto reducido, en concordancia con el desplazamiento apical del centro del resistencia del diente y con el tipo de movimiento a realizar(13), además el TO fue realizado mediante la utilización de arcos de acero de bajo calibre para obtener fuerzas leves e intermitentes que se disipen hacia este periodonto facilitando su reparación entre cada activación ortodóncica(14). El momento de inicio del TO en este caso fue después de 6 meses luego del alta periodontal ya que se considera estrictamente prioritario que se cumplan los siguientes criterios previos al TO: 1) asegurar la adherencia del paciente a este largo tratamiento, 2) eliminar la infección periodontal y alcanzar el nivel de salud periodontal y 3 ) lograr la evidencia clínica de estabilidad periodontal durante el seguimiento en TSP antes de iniciar el TO, a diferencia de lo realizado por Melsen ${ }^{(7)}$ y $\operatorname{Re}^{(10)}$ quienes comenzaron el TO entre el día 10 y 14 luego del TP, quirúrgico o no quirúrgico, quienes buscaban potenciar el TO con la respuesta inflamatoria que se produce durante la cicatrización periodontal ya que las fuerzas ortodóncicas también inducen una respuesta inmunoinflamatoria ${ }^{(15)}$. Radiográficamente la intrusión ortodóncica mostró un mínimo de reabsorción radicular apical externa, con redondeamiento del ápice, sin pérdida de vitalidad pulpar (Figura 5). Para finalizar el tratamiento, la opción del implante dental para reemplazar el diente 1.4 ausente demostró ser un tratamiento rehabilitador predecible. Así, los resultados de este caso clínico muestran una mejora clínica y estabilidad periodontal por al menos 6 años luego de finalizado el TO, manteniendo la dentición natural de la paciente, sin efectos secundarios, sin pérdida dentaria adicional a las que originalmente perdió. Este protocolo pudiese aplicarse a más pacientes que tengan periodontitits y MDP, siempre y cuando cumplan los criterios mencionados anteriormente previos al TO.

\section{RELEVANCIA CLÍNICA}

El tratamiento periodontal y ortodóncico en pacientes con migración dentaria patológica por periodontitis es un desafío ya que involucra el manejo de los tejidos periodontales y el abordaje ortodóncico en 
periodonto reducido. No debe ser un tratamiento estandarizado sino que una biomecánica individualizada, conservador y reteniendo la dentición natural.

Se justifica la presentación de este caso inédito, con el protocolo de la Unidad de Ortodoncia y Periodoncia de la Facultad de Odontología de la Universidad de Chile.
La consecuencia práctica es que puede ser un modelo terapéutico para su aplicación en otros pacientes con similitudes clínicas.

\section{CONFLICTO DE INTERÉS}

Los autores relatan no tener conflicto de interés

\section{Bibliografía}

1. Chasens Al. Periodontal disease, pathologic tooth migration and adult orthodontics. New York J Dent. 1979;49: 40-3.

2. Antoun JS, Mei L, Gibbs K, Farella M. Effect of orthodontic treatment on the periodon-tal tissues. Periodontol 2000. 2017:74(1):140-57.

3. Topkara A, Karaman Al, Kau CH. Apical root resorption caused by orthodontic forces: A brief review and a long-term observation. Eur J Dent. 2012;6(4):445-53.

4. Krishnan V, Davidovitch Z. Cellular, molecular, and tissue-level reactions to orthodon-tic force. Am J Orthod Dentofacial Orthop. 2006;129(4):469.e1-32.

5. Ericsson I, Thilander B, Lindhe J, Okamoto $\mathrm{H}$. The effect of orthodontic tilting move-ments on the periodontal tissues of infected and non-infected dentitions in dogs. J Clin Periodontol. 1977;4(4): 278-93.

6. Melsen $B$. Tissue reaction following application of extrusive and intrusive forces to teeth in adult monkeys. Am J Orthod. 1986:89: 469-75

7. Melsen B., Agerbaek N., Markenstam G. Intrusion of incisors in adult patients with marginal bone loss. Am J Orthod and Dentofacial Orthop. 1989:96(3):232-41.

8. Krishnan V, Davidovitch Z. On a path to unfolding the biological mechanisms of or-thodontic tooth movement. J Dent Res. 2009;88(7):597-608.

9. van Gastel J, Quirynen M, Teughels W, Coucke W, Carels C. Longitudinal changes in microbiology and clinical periodontal variables after placement of fixed orthodontic appliances. J Periodontol. 2008;79(11):2078-86.
10. Re S, Corrente G, Abundo R, Cardaropoli D. Orthodontic treatment in periodontally compromised patients: 12-year report. Int J Periodontics Restorative Dent. 2000;20(1):31-9.

11. Janson M, Janson G, Murillo-Goizueta OE. A modified orthodontic protocol for ad-vanced periodontal disease in Class II division 1 malocclusion. Am J Orthod Dentofa-cial Orthop. 2011;139(4 Suppl):S133-44

12. Tavares CA, Allgayer S, Calvete Eda S, Polido WD. Orthodontic treatment for a patient with advanced periodontal disease: 11-year follow-up. Am J Orthod Dentofacial Or-thop. 2013;144(3):455-65.

13. Kondo T, Hotokezaka H, Hamanaka R, Hashimoto M, Nakano-Tajima T, Arita $\mathrm{K}$, et al. Types of tooth movement, bodily or tipping, do not affect the displacement of the tooth's center of resistance but do affect the alveolar bone resorption. Angle Orthod. 2017;87(4):563-569.

14. Cuoghi OA, Tondelli PM, Mendonça MR, Aiello CA, Costa SC, Tanaka OM. Effect of different types of force on the amount of tooth movement, hyaline areas, and root re-sorption in rats. Eur J Gen Dent. 2018;7:66-71.

15. Zeng M, Kou X, Yang R, Liu D, Wang X, Song Y, et al. Orthodontic Force Induces Sys-temic Inflammatory Monocyte Responses. J Dent Res. 2015;94(9):1295-302. 